\title{
Electron, hole, and exciton spectra in a quantum wire crossing the quantum well
}

\author{
O.M. Makhanets, A.M. Gryschuk, M.V. Tkach \\ Chernivtsi National University \\ 2, Kotsyubinsky str., 58012 Chernivtsi, Ukraine; e-mail: theorphys@chnu.cv.ua
}

\begin{abstract}
The electron, hole, and exciton energy spectra are calculated within the effective mass and rectangular potential approximations for a combined semiconductor nanoheterosystem consisting of a cylindrical semiconductor quantum wire crossing the plane quantum well. It is shown that the electron (hole) in such a system is characterized by five quantum numbers related to five degrees of freedom for a quasiparticle. The dependences of the quasiparticle energy on the quantum wire radius and the quantum well width are researched.
\end{abstract}

Keywords: quantum wire, quantum well, quantum dot, energy spectrum.

Manuscript received 07.02.07; accepted for publication 27.09.07; published online 31.10.07.

\section{Introduction}

The progress in the investigation of low-dimensional semiconductor systems is stimulated by the development of new technologies of the fabrication of nanocrystals which allow one to produce different nanoheterosystems (two-dimensional quantum wells, one-dimensional quantum wires (QW), and zero-dimensional quantum dots (QD) [1, 2]).

In order to obtain the new useful properties of modern objects, it is necessary to study the physical phenomena taking place in them or, in other words, it is necessary to establish the theory for the basic quasiparticles and their interaction between one another and with outer fields of different nature. The theory of the spectra of electrons, holes, excitons, and phonons and the interaction of these quasiparticles between one another and also with the electric and magnetic fields in quantum dots [3-5], quantum wires [6, 7], and quantum wells $[8,9]$ is related to the field of mesophysics. It was intensively developed during the last decades and reached not only the qualitative but also quantitative agreement with the experimental data.

In addition, the rapid development of experimental investigations of nanoheterosystems brought to the existence of rather complicated combined nanoconstructions containing different spatial combinations of quantum dots, quantum wires, and quantum wells. The research of such systems is important due to the perspective of their utilization in the physical, biomedical, and optoelectronic devices [10]. The theory of the spectra of quasiparticles in such systems has not been constructed yet, because they are rather complicated for the mathematical description. Therefore, it is interesting and important to study the peculiarities of the behavior of quasiparticles (electrons, holes, and excitons) at least in rather simple systems. One of them is a cylindrical semiconductor quantum wire crossing the plane quantum well.

\section{Theory of the spectrum of an uncoupled electron- hole pair in a cylindrical quantum wire crossing the quantum well}

Here, we consider the nanoheterosystem consisting of a cylindrical semiconductor quantum wire with radius $\rho_{0}$ crossing the plane quantum well with width $h_{0}$ (Fig. 1).

Depending on the relations between the potentials formed by the media " 0 " $\left(U_{0}\right)$, "1" $\left(U_{1}\right)$, "2" $\left(U_{2}\right)$ for the electron (hole), the crossing region creates a cylindrical quantum dot (when $U_{0}<U_{1}$ and $U_{0}<U_{2}$ ) or an antidot $\left(U_{0}>U_{1}, U_{0}>U_{2}\right)$. The system is placed into the external medium ("3") with the infinite potential $\left(U_{3}\right)$ for a quasiparticle.

The other problem is the scattering at the complicated potential barrier. It is to be considered in future.

In the case where $U_{0}<U_{1}$ and $U_{0}<U_{2}$, we concern the problem of the stationary states of quasiparticles localized in the space of a quantum dot (medium "0"). Due to the circumstance that the theories of the spectra 
of an electron and a hole are developed in analogy, the further consideration is performed for an electron.

In order to study the electron energy states, one has to solve the stationary Schrödinger equation

$\left(-\frac{\hbar^{2}}{2} \vec{\nabla} \frac{1}{m(\vec{r})} \vec{\nabla}+U(\vec{r})\right) \psi(\vec{r})=E \psi(\vec{r})$.

In the cylindrical coordinate system, it takes the form

$\left[-\frac{\hbar^{2}}{2}\left(\frac{1}{\rho} \frac{\partial}{\partial \rho} \rho \frac{1}{\mu(\rho, z)} \frac{\partial}{\partial \rho}+\frac{1}{\rho^{2}} \frac{\partial^{2}}{\partial \varphi^{2}}+\frac{\partial}{\partial z}\right)+U(\rho, z)\right] \times$ $\times \psi(\rho, \varphi, z)=E \psi(\rho, \varphi, z)$.

The effective masses and the potential energies of an electron in different parts of the nanoheterosystem are fixed and assumed to be equal to the corresponding parameters in the respective bulk crystals:

$m(\vec{r})=\left\{\begin{array}{ll}m_{0}, & \text { medium "0" } \\ m_{1}, & \text { medium "1" } \\ m_{2}, & \text { medium "2" }\end{array}\right.$,

$U(\vec{r})=\left\{\begin{array}{ll}U_{0}, & \text { medium "0" } \\ U_{1}, & \text { medium "1" } \\ U_{2}, & \text { medium "2" }\end{array}\right.$.

The dielectric constants in the quantum dot $\left(\varepsilon_{0}\right)$ and in media 1 and $2\left(\varepsilon_{1}, \varepsilon_{2}\right)$ are not very different from each other $\left(\varepsilon_{0} \sim \varepsilon_{1} \sim \varepsilon_{2}\right)$. In this case, the self-forces arising for the charged quasiparticles due to the presence of the interfaces between the cylindrical quantum dot and media 1 and 2 can be evaluated by the following way.

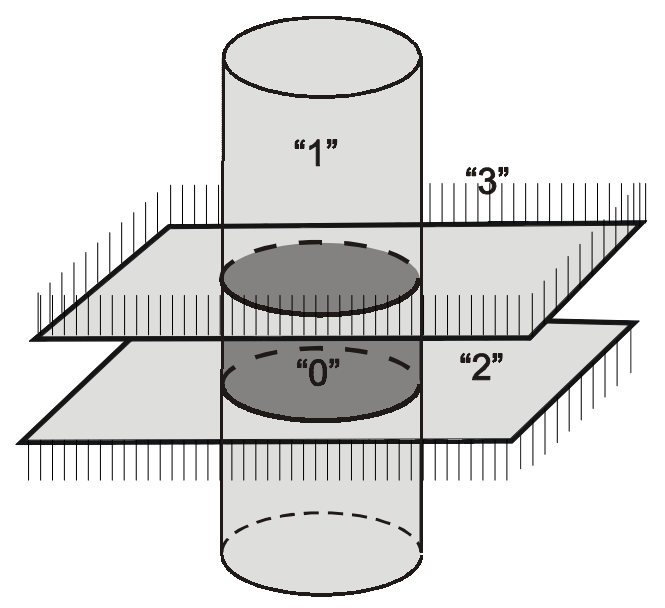

Fig. 1. Geometrical scheme of a quantum wire crossing the quantum well.
The self-force potential of a charged particle in a cylindrical quantum dot is found to be rather complicated. But, taking into the account that the both characteristics of the cylindrical QD are of the same order $\left(\rho_{0} \sim h_{0}\right)$, the cylindrical quantum dot can be approximated by a spherical quantum dot of the same volume. That is why the radius of the quantum dot is $R=\left(\frac{3}{4} \rho_{0}^{2} h_{0}\right)^{1 / 2}$. In order to evaluate the potential of the self-forces of quasiparticles in a spherical quantum dot, one can make use of the fact that, in the deep potential well, a quasiparticle is mainly located at the distance of $R / 2$ from the center of the sphere. Herein, the charged quasiparticle (an electron or hole) in the spherical quantum dot undergoes the action of the polarization field with the potential [11]

$$
U_{p}=\frac{\varepsilon_{0}-\varepsilon_{1,2}}{\varepsilon_{0}+\varepsilon_{1,2}} \frac{e^{2}}{\varepsilon_{0} \sqrt[3]{6 h_{0} \rho_{0}^{2}}}\left[\frac{4}{3}+\ln 3\right] .
$$

Thus, when the geometrical sizes of the cylindrical QD $\left(h_{0}, \rho_{0}\right)$ have the magnitudes satisfying the condition

$U_{\mathrm{p}}<<U_{0}, U_{1}, U_{2}$,

then, the approximation of the rectangular potential (4) can be used to calculate the quasiparticle spectrum.

Taking into account the symmetry of the problem, it is convenient to write the solution of Eq. (1) in the cylindrical coordinate system with the origin at the center of the quantum dot [12] as follows:

$\psi(\vec{r})=\left\{\begin{array}{cc}\psi_{0}(\rho, \varphi, z)=A_{0} J_{m}\left(\chi_{0} \rho\right) e^{i m \varphi}\left(\begin{array}{c}\sin \left(k_{0} z\right) \\ \cos \left(k_{0} z\right)\end{array}\right) \\ \psi_{1}(\rho, \varphi, z) & =A_{1} J_{m}\left(\chi_{1} \rho\right) e^{i m \varphi} e^{-k_{1} z} \\ \psi_{2}(\rho, \varphi, z) & =A_{2} K_{m}\left(\chi_{2} \rho\right) e^{i m \varphi}\left(\begin{array}{c}\sin \left(k_{2} z\right) \\ \cos \left(k_{2} z\right)\end{array}\right)\end{array}\right.$

Here, $A_{0}, A_{1}, A_{2}$ - some constants, $J_{m}(\rho)$ and $K_{m}(\rho)-$ the Bessel and Macdonald functions of integral orders, $m$ - magnetic quantum number,

$$
\begin{aligned}
& \chi_{0}=\sqrt{\frac{2 m_{0}}{\hbar^{2}}\left(E-\frac{\hbar^{2} k_{0}^{2}}{2 m_{0}}\right)}, \\
& k_{1}=\sqrt{\frac{2 m_{1}}{\hbar^{2}}\left(U_{1}-E+\frac{\hbar^{2} \chi_{1}^{2}}{2 m_{1}}\right)}, \\
& \chi_{2}=\sqrt{\frac{2 m_{2}}{\hbar^{2}}\left(U_{1}-E+\frac{\hbar^{2} k_{2}^{2}}{2 m_{2}}\right)},
\end{aligned}
$$

and $k_{0}, \chi_{1}, k_{2}-$ are the unknown parameters. 
Further, we use the continuity conditions for the wave function and its probability current density at all interfaces of the nanoheterosystem,

$$
\begin{aligned}
& \left.\psi_{0}(\rho, \varphi, z)\right|_{\rho=\rho_{0}}=\left.\psi_{2}(\rho, \varphi, z)\right|_{\rho=\rho_{0}} \\
& \left.\frac{1}{m_{0}} \frac{\partial \psi_{0}(\rho, \varphi, z)}{\partial \rho}\right|_{\rho=\rho_{0}}=\left.\frac{1}{m_{2}} \frac{\partial \psi_{2}(\rho, \varphi, z)}{\partial \rho}\right|_{\rho=\rho_{0}}, \\
& \left.\psi_{0}(\rho, \varphi, z)\right|_{z=h_{0} / 2}=\left.\psi_{1}(\rho, \varphi, z)\right|_{z=h_{0} / 2} \\
& \left.\frac{1}{m_{0}} \frac{\partial \psi_{0}(\rho, \varphi, z)}{\partial \rho}\right|_{z=h_{0} / 2}=\left.\frac{1}{m_{1}} \frac{\partial \psi_{1}(\rho, \varphi, z)}{\partial \rho}\right|_{z=h_{0} / 2},
\end{aligned}
$$

and the conditions for the $\psi_{1}$ and $\psi_{2}$ wave functions to be zero at the outer faces of the quantum wire and the quantum well, respectively,

$\left.\psi_{1}(\rho, \varphi, z)\right|_{\rho=\rho_{0}}=0,\left.\quad \psi_{2}(\rho, \varphi, z)\right|_{z=h_{0} / 2}=0$.

As a result, $\chi_{1}, k_{2}$ unknown till now are obtained in the form

$\chi_{1}=\frac{x_{n_{\rho} m}}{\rho_{0}}, \quad k_{2}=\frac{\pi n}{h_{0}}$,

and we get the system of two transcendental equations defining $E$ and $k_{0}$ as

$$
\left\{\begin{array}{c}
\frac{k_{0}}{m_{0}}\left(\begin{array}{c}
\operatorname{ctg}\left(k_{0} \frac{h_{0}}{2}\right) \\
-\operatorname{tg}\left(k_{0} \frac{h_{0}}{2}\right)
\end{array}\right)=-\frac{k_{1}}{m_{1}}, \\
\frac{\chi_{0}}{m_{0}} \frac{J_{m}^{\prime}\left(\chi_{0} \rho_{0}\right)}{J_{m}\left(\chi_{0} \rho_{0}\right)}=\frac{\chi_{2}}{m_{2}} \frac{K_{m}^{\prime}\left(\chi_{2} \rho_{0}\right)}{K_{m}\left(\chi_{2} \rho_{0}\right)} .
\end{array}\right.
$$

Their solutions fix the energies of electron stationary states, $E$. Here, $x_{n_{\rho} m}-$ zeroes of the Bessel functions of integral orders, $n_{\rho}=1,2,3$ - radial quantum number, $n_{z}=1,2,3-$ axial quantum number.

\section{Analysis and discussion of results}

The numerical calculations of the electron (hole) energy spectrum was performed for the nanoheterosystem formed by the semiconductors $\beta-\mathrm{HgS}$ (medium "0") and $\beta-\mathrm{CdS}$ (media "1" and "2"). Their parameters are presented in Table.

\section{Table.}

\begin{tabular}{|c|c|c|c|c|c|c|}
\hline & $m^{e}$ & $m^{h}$ & $\begin{array}{c}U^{e}, \\
\mathrm{eV}\end{array}$ & $\begin{array}{c}U^{h}, \\
\mathrm{eV}\end{array}$ & $a, \AA$ & $\begin{array}{c}E_{g}, \\
\mathrm{eV}\end{array}$ \\
\hline $\mathrm{CdS}$ & 0.2 & 0.7 & 3.8 & 6.3 & 5.818 & 2.5 \\
\hline $\mathrm{HgS}$ & 0.036 & 0.044 & 5.15 & 5.65 & 5.851 & 0.5 \\
\hline
\end{tabular}

In order to study the spectrum of exciton states in the nanoheterosystem under study depending on its geometrical parameters, one has to investigate the spectra of an electron and a hole creating the exciton.

The results of calculations of the electron and hole energies as functions of the quantum wire radius $\left(\rho_{0}\right)$ at the fixed height of the quantum dot, $h_{0}=15 a_{\mathrm{HgS}}$, and the fixed quantum numbers $n_{z}=1, n_{\rho}=1, m=0$ are shown in Fig. 2a and b, respectively.

It is clear that the qualitative behaviors of the electron and hole spectra are the same, while their quantitative difference is caused only by the difference between their effective masses and potential energies. Therefore, we are going to analyze the electron spectrum.

Fig. 2 proves that, with increase in $\rho_{0}$, all quantized energy levels $E_{110 p_{z} p_{\rho}}^{e(h)}$ are shifting into the region of lower energies. At the fixed $n_{z}=1, n_{\rho}=1, m=0$, the spectral levels form the groups over the quantum numbers $p_{z}$ containing the levels with different values of the quantum number $p_{\rho}$. Thus, the ground energy
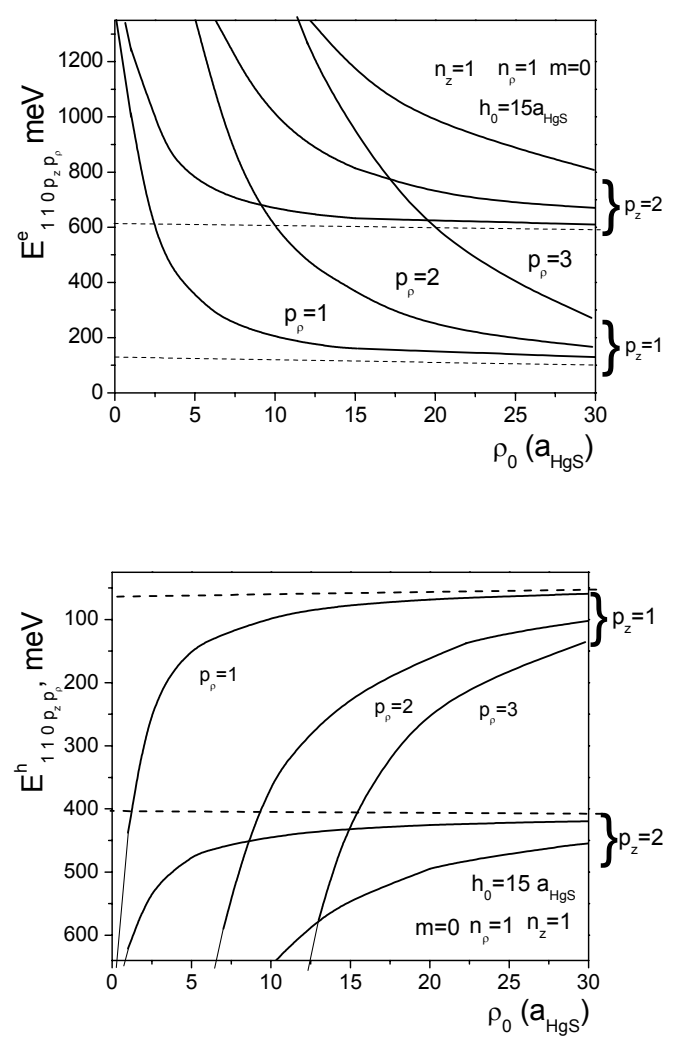

Fig. 2. Electron and hole energy levels $E_{110 p_{z} p_{\rho}}^{e(h)}$ versus the radius of a quantum wire $\left(\rho_{0}\right)$ at the height $h_{0}=15 a_{\mathrm{HgS}}$. 
level has the quantum numbers $n_{z}=1, n_{\rho}=1, m=0$, $p_{z}=1, p_{\rho}=1$. The energies of level groups with $p_{z}=1$ and $p_{z}=2$ decrease with increase in the quantum wire radius $\rho_{0}$ and are limited to the magnitudes of the energies corresponding to two energy states in a semiconductor layer $\beta-\mathrm{HgS}$ placed into the medium $\beta-\mathrm{CdS}$.

The existence of five quantum numbers $n_{z}, n_{\rho}, m$, $p_{z}, p_{\rho}$ is caused by the fact that the electron can be located at the same time in all parts of the nanoheterosystem under study (except region "3") (Fig. 1), and, hence, it is characterized by five degrees of freedom. It is proved by the behavior of the square of the wave function modulus. Its dependence on the coordinates $z$ and $\rho$ is shown in Fig. 3a-d.

Fig. 3a describes the dependence of the square of the wave function modulus of electron ground state on the variables $z$ and $\rho$. It is clear that the probability density has one maximum, as it must be from the considerations of the general theory of localized states of quasiparticles. Fig. $3 b$ corresponds to the first excited energy level with the quantum numbers $n_{z}=1, n_{\rho}=1$, $m=0, p_{z}=1, p_{\rho}=2$. From Fig. 3 , one can see that the probability density has two maxima along the $\rho$ direction and one - along the $z$ axis. Fig. $3 c$ describes the probability density of the electron location at the third
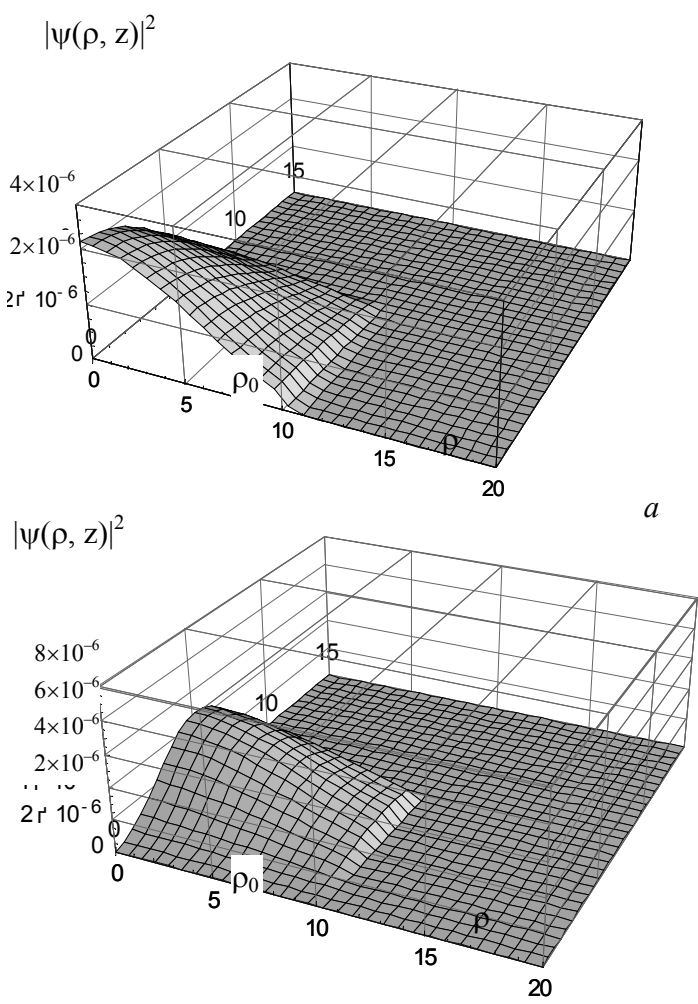

excited level with the quantum numbers $n_{z}=1, n_{\rho}=1$, $m=0, p_{z}=2, p_{\rho}=1$. Herein, the square of the wave function modulus has two maxima along the $z$ axis (the wave function is symmetric, respectively, under the change of $z$ by $-z$ ) and one - along the $\rho$ direction. Finally, Fig. $3 \mathrm{~d}$ corresponds to the quantum numbers $n_{z}=1, n_{\rho}=1, m=0, p_{z}=2, p_{\rho}=2$, and the probability density has two maxima along the both directions.

The general analysis of the system of equations (8) proves that all electron (hole) states are twice degenerated over the magnetic quantum number $m$ (except $m=0$ ). In addition, as one can see from Fig. 2, there is also the casual degeneration of different states (even at $m=0$ ) because the levels with different $p_{z}$ cross one another at the variation of the quantum dot radius $\left(\rho_{0}\right)$.

In Fig. $4 a, b$, we show the dependence of the electron and hole energy levels on the height of the quantum $\operatorname{dot}\left(h_{0}\right)$ at the fixed radius of the quantum wire $\left(\rho_{0}\right)$ and the quantum numbers $n_{z}=1, n_{\rho}=1$, $m=0$.

One can see from Fig. 4 that the behavior of quantized energy levels of an electron and a hole at the increase of the quantum dot height is analogous to that at the increase of the quantum dot radius, but the sequence of energy levels is varied. They now form the groups
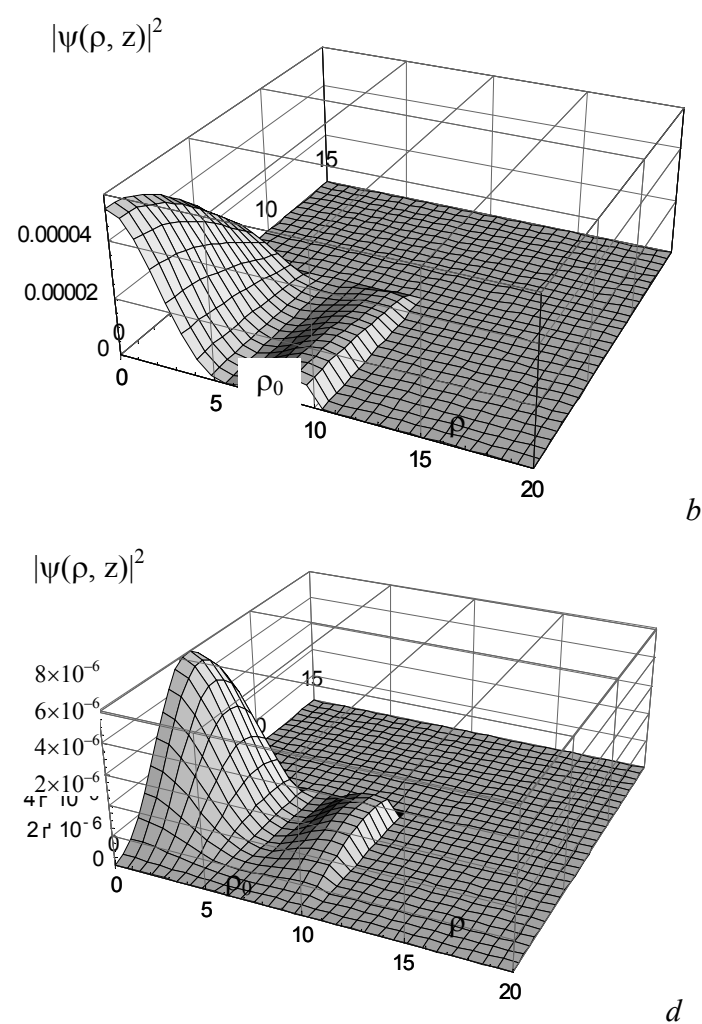

Fig. 3. Dependence of the probability density of the electron location at the ground $(a)$, first $(b)$, third $(c)$, and fourth excited level (d) on the variables $\rho$ and $z$ at $\rho_{0}=10 a_{\mathrm{HgS}}$ and $h_{0}=15 a_{\mathrm{HgS}}$. 

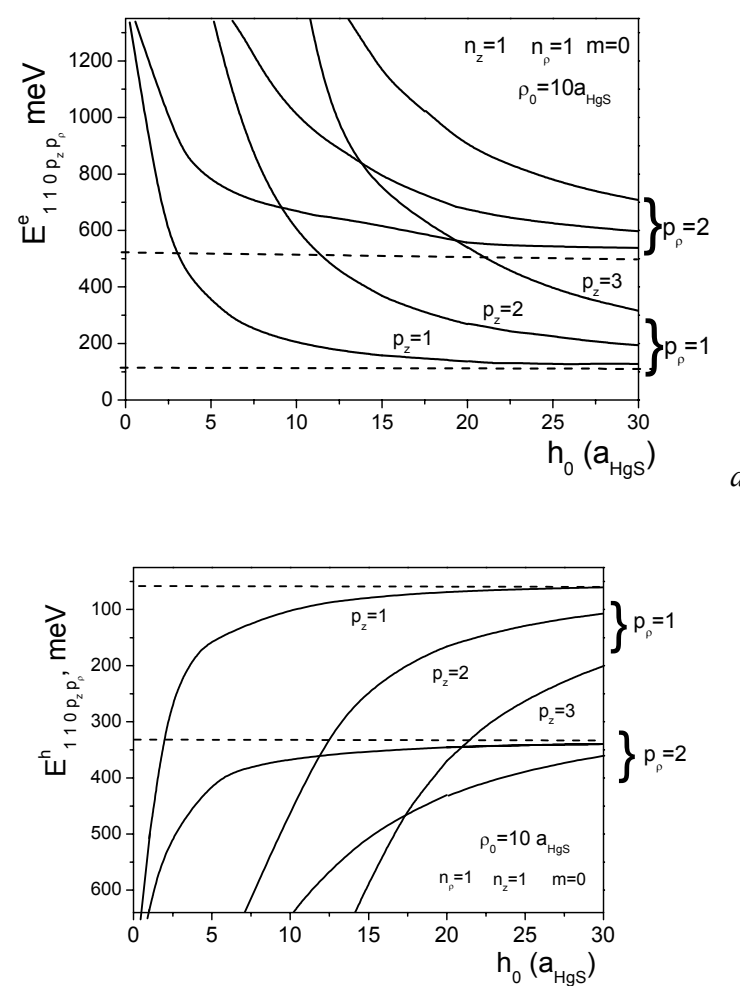

$b$

Fig. 4. Dependence of the electron and hole energy levels $E_{110 p_{z} p_{\rho}}^{e(h)}$ on the height of a quantum $\operatorname{dot}\left(h_{0}\right)$ at $\rho_{0}=10 a_{\mathrm{HgS}}$.

over the quantum number $p_{\rho}$, containing the states with different quantum numbers $p_{z}$. As before, the ground energy level is characterized by the quantum numbers $n_{z}=1, n_{\rho}=1, m=0, p_{z}=1, p_{\rho}=1$. With increase in the height of the quantum dot, the groups of levels with $p_{\rho}=1$ and $p_{\rho}=2$ are limited to the values of energies corresponding to two quantum states of quasiparticles in the quantum wire $\beta-\mathrm{HgS}$ of the radius $\rho_{0}=10 a_{\mathrm{HgS}}$, placed into the medium $\beta-\mathrm{CdS}$.

In Fig. 5a-c, we present the results of calculations of the electron spectrum for four lower energy levels from $p_{z}=1,2, \quad p_{\rho}=1,2, \quad p_{z}=1$ (Fig. 2a) at $m=0$, $m=1, m=2, \rho_{0}=10 a_{\mathrm{HgS}}, h_{0}=15 a_{\mathrm{HgS}}$ in a wide range of quantum numbers $n_{\rho}$ and $n_{z}$.

From the figure, the following main peculiarities of $E_{n_{z} n_{\mathrm{p}} m p_{z} p_{\mathrm{p}}}^{e}$ are obvious.

The increase of the quantum numbers $n_{z}, n_{\rho}, m$ causes the increase of the electron energies in stationary states. Herein, the dependence of the quasiparticle energy on the quantum numbers $n_{\rho}$ and $n_{z}$ is smooth (almost linear), and its dependence on the magnetic quantum number $m$ is sharper (square). So, for example, quantum number $m$ is sharper (square). So, for example, at $m=0$, the stationary states with $p_{z}=1,2, p_{\rho}=1,2$ exist in the nanosystem (Fig. 5a). But, at $m=1$, the state with $p_{z}=2, p_{\rho}=2$ exists only at $n_{\rho}=1$ and $n_{z}=1,2,3$ (Fig. $5 b$ ). When $m=2$, there is no stationary state with $p_{z}=2$.


$b$

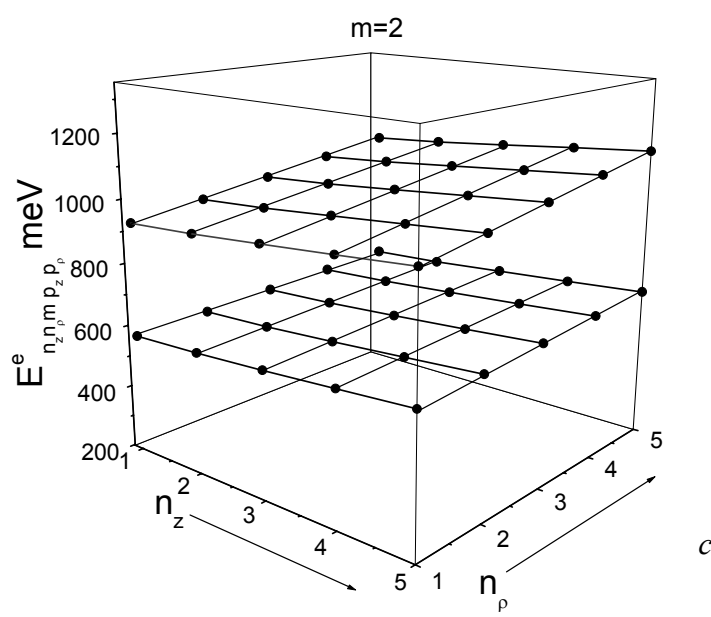

Fig. 5. Dependence of electron energy on the quantum numbers $n_{z}, n_{p}, m$ at $\rho_{0}=10 a_{\mathrm{HgS}}, h_{0}=15 a_{\mathrm{HgS}}$. 
It is worth to note that the information on the electron and hole spectral characteristics does not depict the total picture of radiating or absorbing ability of the system under study. Thus, it is important to consider the problem of the existence of bound states of these quasiparticles or the possibility for an exciton to arise. Let it be „born” in the region of a quantum dot with height $h_{0}$ and radius $\rho_{0}$.

In order to solve this problem, one has to use the Schrödinger equation

$\hat{H}_{e x}\left(\vec{r}_{e}, \vec{r}_{h}\right) \psi_{e x}\left(\vec{r}_{e}, \vec{r}_{h}\right)=E_{e x} \psi_{e x}\left(\vec{r}_{e}, \vec{r}_{h}\right)$

with the Hamiltonian

$\left.\hat{H}_{e x}\left(\vec{r}_{e}, \vec{r}_{h}\right)=\hat{H}_{e}\left(\vec{r}_{e}\right)+\hat{H}_{h}\left(\vec{r}_{h}\right)+U\left(\mid \vec{r}_{e}-\vec{r}_{h}\right) \mid\right)+E_{g 0}$

where $E_{g 0}$ - width of the forbidden band of the quantum dot material, $\hat{H}_{e, h}-$ Hamiltonian of the uncoupled pair of an electron and a hole, and

$U\left(\left|\vec{r}_{e}-\vec{r}_{h}\right|\right)=-\frac{e^{2}}{\varepsilon\left(\vec{r}_{e}, \vec{r}_{h}\right)\left|\vec{r}_{e}-\vec{r}_{h}\right|}$

- electron-hole interaction potential, where $\varepsilon\left(\vec{r}_{e}, \vec{r}_{h}\right)$ dielectric constant which is the complicated function of the displacements of an electron and a hole inside the nanosystem.

Equation (9) cannot be solved exactly. But, taking into the account that the energy of electron-hole interaction is much smaller than the energy of their size quantization, the energy of exciton excitement can be calculated with enough exactness by the formula

$E_{k_{h}}^{k_{e}}=E_{n_{z} n_{\mathrm{\rho}} m p_{z} p_{\mathrm{\rho}}}^{e}+E_{n_{z} n_{\mathrm{\rho}} m p_{z} p_{\rho}}^{h}+E_{g 0}$,

where $k \equiv n_{z} n_{\rho} m p_{z} p_{\rho}$.

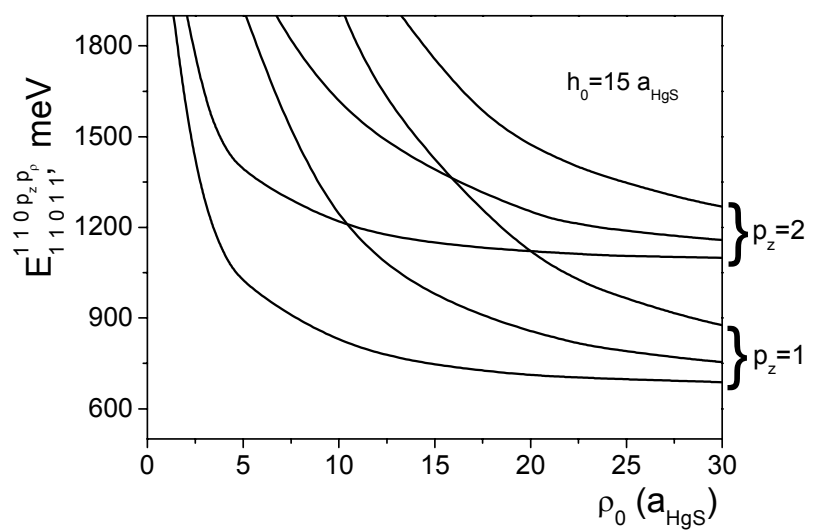

Fig. 6. Dependence of exciton energy $E_{11011}^{110 p_{z} p_{\rho}}$ on the radius of quantum wire $\left(\rho_{0}\right)$ at $h_{0}=15 a_{\mathrm{HgS}}$.
In Fig. 6, we show the dependences of the exciton energies $E_{k_{h}}^{k_{e}}\left(k \equiv n_{z} n_{\rho} m p_{z} p_{\rho}\right)$ of the lower spectrum part on the quantum wire radius at the fixed height of the quantum dot $h_{0}=15 a_{\mathrm{HgS}}$. From the figure, one can see that, with increase in the quantum dot radius, the exciton energies of all states decrease. It is explained by the behavior of $E_{110 p_{z} p_{\rho}}^{e}$ and $E_{110 p_{z} p_{\rho}}^{h}$ analyzed before and their general contribution to the exciton excitement energy (12).

We note that the exciton excitement energy can be located rather deep in the forbidden band of a threedimensional crystal $\beta-\mathrm{CdS}$, and it is very sensitive to the variation of the quantum wire radius what is to be clearly observed in the experiment.

\section{Conclusions}

The results of the performed research allow us to draw the following conclusions.

An electron (hole) in the combined nanoheterosystem under study is characterized by five degrees of freedom, thus its energy spectrum is defined by five quantum numbers $n_{z}, n_{\rho}, m, p_{z}, p_{\rho}$.

At the fixed $n_{z}, n_{\rho}, m$, the spectral levels create the groups over the quantum nuber $p_{z}$, when the radius of quantum wire $\rho_{0}$ is varied, and over the quantum number $p_{\rho}$, when the height of of the quantum dot $h_{0}$ is varied. With increase in $\rho_{0}$ or $h_{0}$, all quantized energy levels shift into the region of smaller energies limited by the bottom of the quantum well.

The increase in the magnitude of the quantum numbers $n_{z}, n_{\rho}, m$ causes the increase in the electron energies in the stationary states. Herein, the dependence of the quasiparticle energy on the quantum numbers $n_{\rho}$ and $n_{z}$ is smooth (almost linear) and its dependence on the magnetic quantum number $m$ is sharper (square).

At the decrease of the quantum wire radius, the exciton energies in all states increase and can be located rather deep in the forbidden band of the bulk crystal $\beta-\mathrm{CdS}$.

References

1. Zh.I. Alferov // Fiz. Tekh. Poluprov. 32, No 1, p. 3 (1998) (in Russian).

2. N.N. Ledentsov, V.M. Ustinov, V.A. Schukin, P.S. Kop'ev, Zh.I. Alferov, D. Bimberg // Fiz. Tekh. Poluprov. 32, No 4, p. 385 (1998) (in Russian).

3. N.V. Tkach // Fiz. Tverd. Tela 39, p. 1109 (1997) (in Russian).

4. Ch. Greus, R. Spiegel, P.A. Knipp, T.L. Reinecke, F. Faller, A. Forchel // Phys. Rev. B49, p. 5753 (1994). 
5. D. Schooss, A. Mews, A. Eychmuller, H. Weller // Phys. Rev. B49, p. 17072 (1994).

6. X.F. Wang, X.L. Lei // Phys. Rev. B49, p. 4780 (1994).

7. N.C. Constantinou, B.K. Ridley // Phys. Rev. B41, p. 10627 (1990).

8. N. Mori, T. Ando // Phys. Rev. B40, p. 6175 (1989).
9. G.Q. Hai, F.M. Peeters, J.T. Devreese // Phys. Rev. B48, p. 4666 (1993).

10. R.W. Siegel // Proc. E-MRS 24 (2004).

11. N.A. Efremov, S.I. Pokutnii // Fiz. Tverd. Tela 27, p. 48 (1985) (in Russian).

12. N.V. Tkach, A.M. Makhanets // Fiz. Tverd. Tela 47, p. 550 (2005) (in Russian). 